\title{
Interstadiale Bodenbildungen in oberitalienischen Würm-Lössen
}

\author{
Von Otto Fränzle, Bonn
}

\author{
Mit 1 Abbildung im Text
}

$\mathrm{Z}$ us a mmenf a s sung. Es wird der Versuch unternommen, auf der Grundlage von Geländebeobachtungen und Literaturangaben eine Karte der mutmaßlichen Lößverbreitung in Oberitalien zu entwerfen. Dann werden fünf Interstadialböden beschrieben. Im Dora Ripária-Gebiet westlich Turin liegen zwei Würm!össe über dem letztinterglazialen, solifluidal umgelagerten Plastosol. Der untere ist zu einer Braunerde-Parabraunerde, der obere zu einer extrem sauren Parabraunerde verwittert. Auch der Löß auf den Rißmoränen des Dora Báltea-Gletschers gehört zwei Würmphasen an. Der darauf entstandene interstadiale und rezente Boden sind beides extreme Pseudogleye. Der fossile Boden ist reich an ehemaligen Frostspalten. Am Tessin konnte auf den Deckenschottern Riß- und Würmlöß gefunden werden. Der extrem pseudovergleyte Rißlöß ist in noch weit höherem Maße als der zu einer mäßig pseudovergleyten Braunerde verwitterte basale Würmlöß von einem Gitterwerk ehemaliger Frostspalten durchsetzt. Aus dem westlichen Gardasee-Gebiet werden zwei Solifluktionslösse über Rißmoräne beschrieben, von denen der untere zu einer Parabraunerde, der obere zu einer Parabraunerde-Braunerde verwitterte. Östlich des Gardasees wurde eine interstadiale Schwarzerdebildung angetroffen, die später zusammengeschwemmt und von Moränen überlagert wurde. Abschließend werden das Bildungsklima der fossilen Böden und ihre stratigraphische Einordnung diskutiert.

A bstract. Based on field-work and previous publications an attempt is made to outline the distribution of loess in Northern Italy. Five interstadial soils are described. In the Dora Ripária region W of Torino two Würm loesses overly the soliflually eroded plastosol of the Last Interglacial. The lower one weathered to a "sol brun lessivé", the upper to an extremely acid "sol lessivéc. Also the loess on the Riss moraines of the Dora Báltea glacier was deposited in two phases of the Würm period. The (interstadial and recent) soils developed therefrom, are both extreme pseudogleys, the first of which is rich in former frost wedges. The Mindel gravels of the river Ticino are covered by both Riss and Würm loesses. The extremely pseudogleyed Riss loess has by far more and bigger frost wedges than the older Würm loess, which is weathered into a moderately pseudogleyed braunerde. Two soliflual loesses covering Riss moraines are described from the western Garda region; the lower weathered into a "sol lessivé", the upper into a "sol brun lessivéc. E of the lake of Garda an interstadial black earth was found, which had been washed into a valley and then covered by Würm moraines. Finally the developmental climate and the stratigraphic position of the described fossil soils are discussed.

Für die Feinstratigraphie des oberen Pleistozäns und insbesondere der Würmeiszeit spielen durch Bodenhorizonte gegliederte Lößprofile eine sehr wesentliche Rolle. Dies gilt vor allem für jene Gebiete, wo organische Bildungen für eine relative oder absolute Altersbestimmung nicht oder nur in unzureichendem Umfange zur Verfügung stehen. Außerdem gestatten fossile Böden in vielen Fällen wichtige Rückschlüsse auf das Klima ihrer Entstehungszeit.

Während nun die diesbezüglichen Forschungen in Mittel- und Westeuropa schon weit vorangetrieben sind und für viele Gebiete eine mehr oder weniger detaillierte Regionalgliederung vorliegt und eines der wesentlichsten Anliegen in der stratigraphischen Einstufung neu hinzukommender Einzelprofile sowie ihrer paläoklimatischen Ausdeutung besteht, ist unsere Kenntnis der Lößstraten Südeuropas um vieles lückenhafter. Auch das im folgenden näher zu behandelnde Beispiel Norditaliens (zwischen Dora Ripária und Etsch) zeigt, daß selbst über grundlegende Dinge wie die Lößverbreitung noch weitgehend Unklarheit herrscht.

Wenn man von dem klassischen Lößvorkommen von Rívoli-Turin und dem ebenfalls schon sehr lange bekannten, aber nicht von allen als Löß aufgefaßten „Volpino“ östlich des Gardasees absieht, findet man Löß nur sehr vereinzelt im Rahmen allgemein gehaltener Profilbeschreibungen erwähnt. So führen ibn $\mathrm{S}_{\mathrm{ACCO}}$ (1886) aus der Umgebung von Fossano-Carrù (60 km südlich Turin), PENCK (1909) von Carpenédolo, Levy (1914/15) von der Baraggia di Candelo und BECK (1938) von der Ferretto-Hochfläche südlich Varese 
an. Lößartigen, hellen Sand beschreiben LEvy (a. a. O.) und NANGERoni (1954a) als Deckschicht von Würmmoränen des Tessin-Gletschers. Echten Löß auf Hochterrassenschottern bzw. Rißmoränen des Tessingletschers bildet NANGERONI (1954b) unter der Bezeichnung „löss argilloso" bzw. "löss-lehm“ ab. VENzo (1952, 1953) erwähnt Löß im Zusammenhang mit der Fauna von Sambughetto im Strona-Tal und aus dem Gebies des Adda-Gletschers bei Calusco. Häufiger als die Nennung von Löß sind Angaben übs:r die Lehmbedeckung von Altmoränen, Ferretto oder Hochterrassenschottern ohne Hinwєis auf Zusammensetzung und Bildungsgeschichte dieses Lehms, so daß sich nicht entscheiden läßt, ob hier eine interglaziale Verwitterungsrinde oder ein verlehmter Löß vorliegt. Inş̧esamt läßt sich wohl sagen, daß in Oberitalien bisher dem Löß im Vergleich zu den anderen Glazialablagerungen, vorab den Moränen, leider sehr wenig Beachtung geschenkt wurde.

Schon die erste Begehung des Gardasee-Moränenamphitheaters zeigte, daß hier Löß eine weit größere Ausdehnung besitzt, als auf Grund der bislang vorliegenden Literatur zu vermuten war. Die sich anschließenden Detailuntersuchungen, die im vergangenen Jahr im Gletschergebiet der Dora Ripária, im Pellice-T'al (40 km südwestlich Turin), in den Walliser Alpen und den Amphitheatern des Dora Báltea-, Tessin-, Comer-See- und Gardasee-Gletschers durchgeführt wurden, zeigten, daß das Gleiche in der ganzen nördlichen Poebene und am Südrand der Alpen der Fall ist (das höchste beobachtete Lößvorkommen liegt in $1500 \mathrm{~m}$ Meereshöhe und entspricht damit möglicherweise den von SCHönhals in den Zentralalpen beobachteten). Auch der Lehm der älteren Autoren erwies sich in der Regel als Lößboden über den jeweiligen mehr oder weniger abgetragenen interglazialen Plastosolen, die schon früher kurz beschrieben wurden (FRÄNzLE 1959). Die mir bekannt gewordenen Lößareale sind auf der beigefügten Ubersichtskarte (Abb. 1) wiedergegeben.

\section{A. Die Interstadialböden im einzelnen}

Mehrfach wurden in den letzteiszeitlichen Lössen fossile Bodenbildungen beobachtet, deren wichtigste im folgenden beschrieben werden (auf der Karte sind die Profile mit (1) usf. gekennzeichnet).

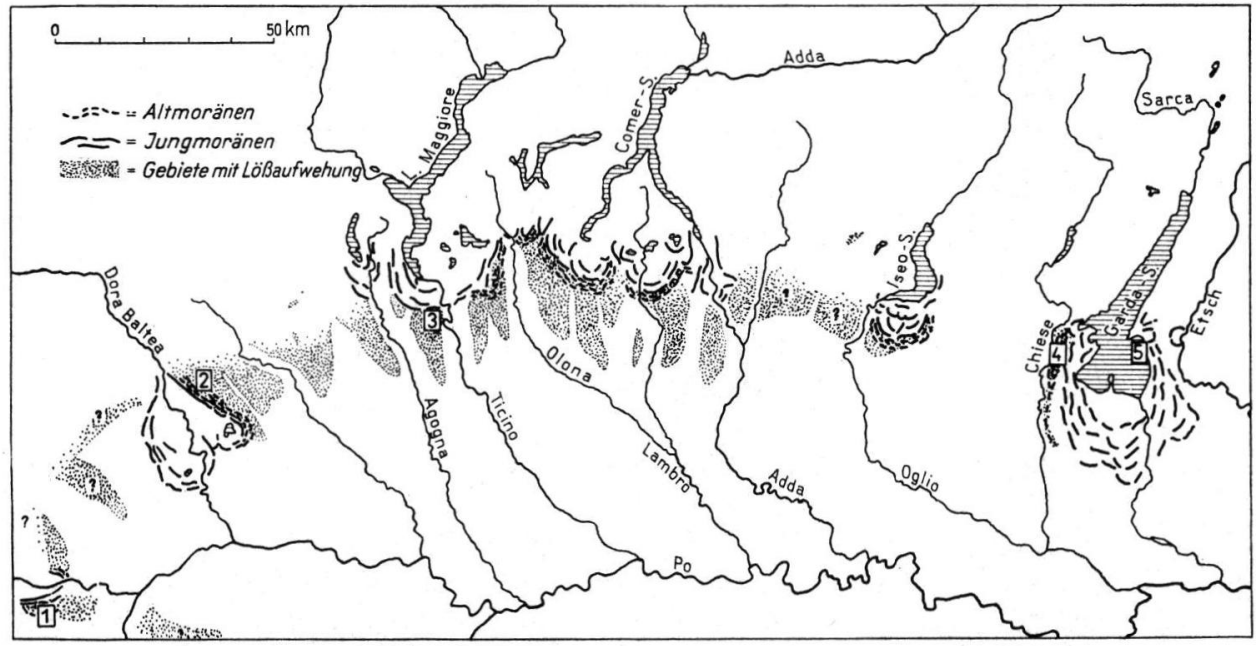

Abb. 1. Kartenskizze der Gebiete mit nachgewiesener und vermuteter (?) Lößaufwehung. Geologisch-morphologische Kartengrundlagen: PENCK \& Brückner (1909, S. 786/787), Atlante Fisico-Economico d' Italia del T.C.I., Waldbaur (1958). Mensching (1954). Von den singulären Vorkommen innerhalb der Alpen abgesehen, sind es im wesentlichen die prä-würmeiszeitlichen Quartäraufragungen der Poebene, die Löß(lehm) tragen. Die Begrenzung der Lößareale folgt diesen schematisch. 
1) Das Gebiet des Dora Ripária-Gletschers

Der südlichste Gletscher Piemonts, der das Alpenvorland erreicht hat, war der der Dora Ripária. Er hinterließ ein sehr eindrucksvolles Moränenamphitheater, dessen glazigene Natur erstmals von MARTins und GASTALDI im Jahre 1850 erkannt worden ist.

Die hydrographisch rechte Seite des Zungenbeckens wird von drei sich zangenförmig nach innen krümmenden Seiten- bzw. Endmoränensystemen begrenzt. Der äußerste Moränenwall beginnt südöstlich des Moncuni, eines dem Alpenrand vorgelagerten $641 \mathrm{~m}$ hohen Berges, und zieht sich mit mannigfachen Unterbrechungen auf Rívoli zu. Ungleich eindrucksvoller ist der mittlere, der Hauptendmoränenzug, der sich an die Nordflanke des Moncuni anlehnt und leicht geschwungen bis nach Rívoli hinzieht. Auch er ist mehrfach durch Schmelzwassertäler gegliedert und oft in einzelne Rücken aufgelöst. An ihn angeschmiegt ist der innerste Moränenzug, der gleichfalls an der Nordflanke des Moncuni beginnt, und der sich zunächst geschlossen bis zum Dörfchen Rosta hinzieht. Hier trennt er sich von dem höheren Rívolizug ab und biegt nach NE zur Dora Ripária hin, die er bei Alpignano erreicht. Von der Moränendurchsägung wendet er sich nach W zum Gebirgsrand zurück. An seine Außenseite lehnt sich ein älterer Zug an, der augenscheinlich das Aquivalent des allerdings sehr viel eindrucksvolleren Hauptzuges von Rívoli darstellt.

Der Formengegensatz zwischen dem südlichsten und dem Hauptendmoränenwall von Rívoli ist sehr auffällig. Die Rívoli-Moräne, von MEnsching (1954) auf Grund morphometrischer Untersuchungen ins Jungriß gestellt, zeigt relativ frische Formen, während die südlichste, von PencK (1909) als einzige ins Riß gestellt und von Mensching (a. a. O.) als Altriß aufgefaßt, vergleichsweise verwaschen ist.

Die maximal 10 Meter mächtige Lößdecke der Altmoränen wurde m. W. zuerst von Viglino \& CAPEDER (1898) beschrieben; der mit ihr wohl im Zusammenhang stehende Löß von Turin erfuhr schon 1887 durch $S_{\Lambda C C O}$ eine gute Darstellung. PENCK (1909) sah den Löß auf Grund seiner unzutreffenden Einstufung der Rívoli-Moräne als postglazial an, eine Meinung, die sich bekanntlich bis zur Neuuntersuchung durch Menschurng (a.aO.) hielt. Das Vorkommen ist nicht nur wegen seiner Ausdehnung und Mächtigkeit, sondern auch wegen seiner pedologischen Gegebenheiten von hohem Interesse.

In einem Lößhohlweg, der zum Hauptmoränenzug hinführt, wurde ein Profil freigelegt, das schon makroskopisch eine Zweischichtigkeit (im sedimentologischen Sinne) erkennen läßt. Dem entspricht auch die bodentypologische Differenzierung. Die obersten $140 \mathrm{~cm}$ des von siliciphilen Edelkastanien bestandenen Lößlehms stellen eine Parabraunerde mit den Subhorizonten $\mathrm{A}_{1}, \mathrm{~A}_{3}, \mathrm{~B},(\mathrm{~B}) / \mathrm{C}, \mathrm{Ca} / \mathrm{C}$ dar, die sich besonders durch ihren abnorm tiefen $\mathrm{pH}$-Wert von 3,6 (in $\mathrm{KCl}$-Suspension) im $\mathrm{A}_{3}$ auszeichnet. Der basale Kalkanreicherungshorizont tritt auch morphologisch als kleine Vorkragung in der Wand des Hohlweges in Erscheinung.

Der basale Löß $(1 \mathrm{~m})$ ist $\mathrm{zu}$ einer Braunerde-Parabraunerde $\left(f A_{3}, f(B) / B, f(B) / C\right)$ verwittert, die im oberen Profilteil sekundär aufgekalkt ist. Unterlagert wird er von einer Fließerde, die in einem benachbarten Aufschluß erstmals von Mensching (1954) erkannt wurde; darunter folgt verwittertes Rißmaterial, bodentypologisch der (B)- bzw. (B)/C-Horizont eines rubefizierten Plastosols.

$\mathrm{Da}$ der Löß in seiner Gesamtheit ein Produkt der letzten Eiszeit ist, muß die Bildung des fossilen Bodens in einem Interstadial erfolgt sein. Wie aus der geringeren Entwicklungstiefe und allgemeinen Profildifferenzierung zu ersehen, war die Verwitterungs.intensität erheblich geringer als im Postglazial.

Mächtige Lößablagerungen, die unter der Bezeichnung Lehm eine kurze Erwähnung bei SACCO (1887) und bei PENCK (1909) fanden, liegen auf der linken Hochterrasse und dem damit in Zusammenhang stehenden Altmoränengebiet der Dora Ripária. Auch hier sind auf der Terrasse zwei Böden entwickelt, von denen der obere in etwa der hangenden 
Parabraunerde des Lößprofils von Rívoli entspricht. Im Gegensatz zu jenem Profil folgt darunter aber ein sehr ausgeprägter Pseudogley.

2) Das Gebiet des Dora Báltea-Gletschers

Sehr viel ausgedehnter als das eben skizizierte Amphitheater des Dora Ripária-Gletschers ist das der Dora Báltea, das sich hufeisenförmig um Ivrea herumlegt. Das bis auf die Seen von Viverone und Cándia vom Baltea-Fluß eingeebnete Zungenbecken wird von Jungmoränen eingefaßt, deren zahlreiche bogenförmige Krümmungen im Osten eine starke Zerlappung des Gletscherrandes anzeigen (PENCK 1909). Im NE erreichen sie in dem gigantischen Doppelwall der Serra mit 200 bis 250 Metern ihre größte relative Höhe. Das sanft gewellte Hinterland der Serra besteht aus Riß-Moränen, die tiefgründig verwitterten Mindel-Moränen aufliegen. Wie die Altmoränen des Dora Ripária-Gebietes tragen auch sie eine mehrere Meter mächtige Deckschicht letzteiszeitlichen Lösses. In einem Lößhohlweg wurde an der Straße Zubiena-Broglina folgendes Profil aufgegraben:

Bodentypologische Bezeichnung: Pseudogley

Profilaufbau:

$\mathrm{A}_{0-1} \quad 0-5 \mathrm{~cm}$ braunschwarzer Moder;

$\mathrm{A}_{2} \mathrm{~g} \quad 5-10 \mathrm{~cm}$ gelblich-fahlgrauer, krümeliger feinsandiger Lehm, nach unten $\mathrm{zu}$ mit einzelnen Rostflecken und verschmierenden Konkretionen;

g1 10-40 cm gelblich-grauer (10 YR 5/6 - 2,5 Y 6/4) feinsandiger Lehm von mäßig gut ausgebildetem Polyedergefüge mit relativ wenigen Rostflecken und verschmierenden kleinen Konkretionen;

g2 $40-80 \mathrm{~cm}$ bräunlich-gelber (2,5 Y 6/4) feinsandiger Lehm von polyedrsschem Gefüge mit Rostflecken und vielen, z. T. mehr als $1 \mathrm{~cm} \phi$ erreichenden Eisen- und Manganoxydhydrat-Konkretionen, die nach unten $\mathrm{zu}$ immer dichter zusammentreten, um sich im

$\mathrm{g}_{3} \quad 80-90 \mathrm{~cm}$, dem Subhorizont stärkster Konkretionsbildung, zu einer beinahe wabenartig durchlöcherten harten Schicht zusammenzuschließen, an der die Durchwurzelung endet;

$\mathrm{fg}_{1} \quad 90-120 \mathrm{~cm}$ brauner (10 YR 5/6) feinsandiger Lehm von Plattengefüge, das beim Drücken in kleine Polyeder (Sekundärgefüge) zerfällt; Konkretionen sind verhältnismäßig selten; charakteristisch für diesen und den folgenden fossilen Subhorizont sind sehr zahlreiche \pm horizontale fahlgraue (2,5 Y - 5 Y 6/1) Bleichungszonen, die bei einer mittleren Dicke von $0,5 \mathrm{~cm}$ einen Abstand von $1-2 \mathrm{~cm}$ haben;

$\mathrm{fg}_{2} \quad 120-160 \mathrm{~cm}$, wie $\mathrm{fg}_{1}$, aber mit zahlreichen Eisen- und Manganoxydhydratkonkretionen, die von maximal $1 \mathrm{~cm}$ messenden, rostbraunen Höfen umgeben sind und mehreren maximal $2 \mathrm{~cm}$ breiten \pm senkrecht stehenden Bleichungszonen, die sich mit den horizontal verlaufenden zu einem Netzwerk vergittern.

Auffällig sind im fossilen und dem Unterteil des rezenten Bodens vereinzelte kleine ziegelrote Konkretionen, die wohl Einwehungsprodukte präwürmeiszeitlicher rubefizierter Böden darstellen.

Der fossile extreme 'seudogley gehört zweifellos einem Würm-Interstadial an, denn der Löß, aus dem er ent kand, lagert dem letztinterglazialen Braunlehm auf. Die horizontalen und vertikalen $\mathrm{B}^{1}$ sichungszonen sind primär als Frostspalten angelegt worden und dienten in der Folgezeit der interstadialen Pseudovergleyung der Eisen- und Manganverlagerung. Auf den Hängen ist dieser ältere Würmlöß weiterhin der solifluidalen Abtragung zum Opfer gefallen, die auch wesentliche Teile des unterlagernden Interglazialbodens miterfaßt hat. Der jüngere Würmlöß, auf selbst sehr flachen Hängen in Form einer oftmals mehr als ein Meter mächtigen Fließerde ausgebildet, entwickelte sich, wenn kein Wasserstau vorlag, zu einer Braunerde bzw. Parabraunerde, die in Süd-Exposition einen schwach rötlichen Farbton hat.

3) Riß - und W ïrmlöß a m Tessin

An das Moränenamphitheater des Lago Maggiore schließt sich tessinabwärts ein Terrassensystem an, das wegen seiner wundervollen Ausprägung schon frühzeitig erkannt worden ist. Insgesamt lassen sich mehrere Stufen in den Niederterrassenschottern, sodann 
in verhältnismäßig weitem Abstand vom Fluß die Hochterrassenschotter erkennen, die auf der piemontesischen Seite von der Deckenschotterplatte von Divignano überragt werden. Wie die entsprechenden Altmoränen, so überzieht Löß auch die älteren Terrassen des Tessins als mehr oder minder geschlossene Decke. Eine genauere Untersuchung zeigte, daß die Hochterrasse von e in e m Löß, der Deckenschotter hingegen von $z$ w e i Lössen bedeckt ist. PENCK (1909) hat wohl sehr klar den unterschiedlichen Verwitterungsgrad der Nieder- und der Hochterrassenschotter erkannt; die äolische Natur der Ablagerungen auf letzteren ist ihm aber offenbar nicht bewußt geworden. Er schreibt lediglich, daß auf der Hochterrasse ein in der Regel ziemlich mächtiger brauner Lehm liegt, der zu Ziegeln verarbeitet werden kann; bei dem Deckenschotter erwähnt er nur die starke Ferrettisierung.

Der beste mir bekannt gewordene Aufschluß, der die Überlagerung des Deckenschotters mit zu verschiedenen Eiszeiten gehörenden Lössen zeigt, liegt in der aufgelassenen Lehmgrube der Fornace Colombo; die dort angetroffenen Bodenverhältnisse schildert die folgende Profilbeschreibung:

Unter einer aus Gräsern und Sträuchern von Robinia psendoacacia und Castanea sativa bestehenden Vegetation folgen drei Böden, von denen der rezente und der obere fossile, aus Wiirmlöß entstandene Boden schwach bis mäßig pseudovergleyte Braunerden sind, während der untere fossile, aus Rißlöß gebildete Boden einen extremen Pseudogley darstellt. Im einzelnen ist der Profilaufbau wie folgt:

A $\quad 0-10 \mathrm{~cm}$ grauer (10 YR 4/4), humoser, feinsandiger Lehm von Krümelgefüge;

$\mathrm{g} /$ (B) $10-45 \mathrm{~cm}$ brauner, feinsandiger Lehm von Kohärentgefüge, das beim Drücken in subanguläre Bröckel zerfällt; neben verschmierenden Rostflecken treten auch rostbraune Konkretionen auf, die teilweise tiefschwarze (Mn-) Konkretionen umschließen;

fA $\quad 45-55 \mathrm{~cm}$ grauer (10 YR $3 / 3-2,5$ Y $4 / 4$ ) humoser, feinsandiger Lehm von Krümelbis Bröckelgefüge;

$\mathrm{fg} /(\mathrm{B})$ 55-70 $\mathrm{cm}$ brauner (10 YR 5/6), feinsandiger Lehm, der in seinen pedologischen Eigenschaften dem rezenten Boden gleicht, nur daß das Fleckenbild erheblich intensiver wird; pedogenetisch und damit paläoklimatisch aufschlußreich ist, daß dieser - nur in einer flachen Rinne ausgebildete - Boden von einem Gitter feiner horizonta'er und dickerer vertikaler Bleichzonen, die wohl auf Frostspalten zurückgehen, durchzogen ist, und daß er ferner einen hohen Prozentsatz des unterlagernden, stark verwitterten und verfestigten Lösses in Form grober, kantengerundeter Bruchstücke enthält (Fließlöß);

$\mathrm{fg}_{1} \quad 70-120 \mathrm{~cm}$ graubrauner (10 YR 4/4) feinsandiger, außerordentlich stark pseudovergleyter Lehm, der besonders im oberen Teil von zahlreichen horizonta'en und einigen breiten senkrechten Bleichzonen (5 Y 6/3) durchzogen ist;

$\mathrm{fg}_{2} \quad 120-160 \mathrm{~cm}$ in diesem Subhorizont, der in seinen petrographischen Eigenschaften dem vorigen entspricht, sind die mehrere Zentimeter breiten fahlgrauen Bleichungszonen weitabständig und vorwiegend senkrecht verlaufend; sie bilden ein polygonales Netzwerk und gehen wahrscheinlich auf Frostspalten zurück; die durch sie gegeneinander abgegrenzten proßprismatischen, stumpfkantigen Gefügeelemente sind fester a's die Bleichschichten und stets von einer peripheren Rostschicht umgeben;

$\mathrm{fg}_{3} \quad 260-460 \mathrm{~cm}$ brauner, feinsandiger Lehm mit zahlreichen Rostflecken; das kennzeichnende plattige Gefüge dieses Subhorizontes kommt durch die vorwiegend horizontale Anordnung ausgedehnter Eisen- und Manganoxydhydrat-Konkretionen zustande.

Daß Rißalter des untersten Lösses folgt aus seiner strengen Bindung an die Deckenschotterplatten, die ganz ungewöhnliche Entwicklungstiefe des Bodens $(>4 \mathrm{~m})$, die sich bis zur regelrechten Schwartenbildung steigernde Konkretionierung, sowie das Ausmaß der Bleichung. Letztere erreicht auch bei den stärkst pseudovergleyten Würmlössen nie mehr als einen halben Meter, während hier die einzelnen Bleichzonen sich stellenweise zu einer maximal zwei Meter mächtigen Bleichschicht zusammenschließen (Stagnogley). Die ebenfalls bei Würmlössen nie angetroffene außerordentliche Verhärtung wurde oben schon erwähnt. Der Härteunterschiẹd zwischen den Bleichzonen und den großen Prismen bewirkt in Aufschlußwänden, diz längc*: Zcit frai licgen, ein Hzrauspräparieren der Primär- 
spalten durch Herauswittern der Bleichschicht, ebenfalls ein Vorgang, der wegen der fehlenden Verhärtung des Würmlösses nicht auftreten kann.

Daß dieser Rißlöß ungeachtet seiner weiten Verbreitung auch in anderen Teilen der Po-Ebene $\mathrm{m}$. W. bisher nicht als solcher erkannt wurde, beruht wohl auf der mit außerordentlicher Verhärtung einhergehenden Pseudovergleyung, durch die er im Gelände ohne genauere Prüfung durchaus mit stark pseudovergleytem Ferretto verwechselt werden kann. Unter diesen Umständen bedarf es noch eingehender Untersuchungen, inwieweit auch die übrigen Ferretto-Flächen eine Decke von Rißlöß tragen. Dies ist mit Sicherheit der Fall in der Brianza und wahrscheinlich östlich der Adda bei Calusco.

Insgesamt läßt sich aus der Aufeinanderfolge und den Eigenschaften der oben beschriebenen Böden folgende Entwicklungsgeschichte ableiten:

1. Uberdeckung des $M / R$-interglazialen Ferretto mit Rißlöß.

2. Bildung tiefreichender Frostspalten im Löß (Tundrenboden).

3. Außerordentlich intensive Pseudovergleyung im letzten Interglazial, bei der die ehemaligen Frostspalten als Leitbahnen der Reduktion wirkten. Vom Rande der Terrasse her Bildung von Wasserrissen.

4. Solifluktion im Würm-Frühglazial, der sich alsbald die Sedimentation des nur noch als Füllung der kleinen solifluidal ausgestalteten Rinnen erhaltenen älteren Würmlösses überlagerte, wobei Brocken des verhärteten, intensiv pseudovergleyten Rißlösses als „Schutt" verfrachtet wurden. Bildung eines Tundrennaßbodens mit Frostspalten, der nach oben in wenig pseudovergleyten Fließlöß übergeht.

5. Umprägung dieses Fließlösses zu einer Pseudogley-Braunerde im Interstadial.

6. Sedimentation des jüngeren Würmlösses.

7. Bildung des spätglazial-holozänen Bodens.

4) und 5) Das Gebiet des Gardasee-Gletschers

Der Gardasee wird in seinem Südteil von dem größten und stratigraphisch differenziertesten Moränenamphitheater der ganzen Südalpen umschlossen. Die das heutige Relief bestimmenden, vielfach gestaffelten End- und Seitenmoränen gehören alle ins Würm mit Ausnahme der flachen und verwaschenen Züge am Fiume Chiese im Westteil des Moränengebietes (Fränzle 1959, 1960). Diese und der Monte Moscal im Osten des Amphitheaters, an den sich die dortigen äußersten Jungmoränen anlagern, tragen eine Lößdecke, die im

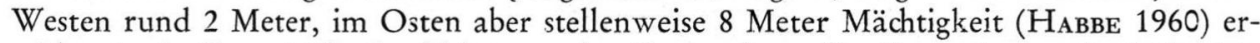
reicht. Auch dieser Löß, der bisher nur im Osten, dort allerdings schon recht frühzeitig (NrColis 1895) erkannt wurde, zeigt sehr intensive Spuren interstadialer Bodenbildungen, die im folgenden, von W nach $\mathrm{E}$ fortschreitend, beschrieben werden.

Ein Wegeinschnitt am Rande des Dorfes Mocasina am F. Chiese zeigt zwei Solifluktionslösse über einer sehr blockreichen, rund $40 \mathrm{~cm}$ mächtigen frühglazialen Fließerde, deren Matrix aus umgelagertem Material des unterlagernden letztinterglazialen Braunlehms besteht.

Der liegende Löß ist rund $60 \mathrm{~cm}$ mächtig. Sein auffälligstes makroskopisches Texturmerkmal ist die (rezente) Auflösung durch große, breitklaffende Trockenrisse, die auf der schrägen Aufschlußwand ein weitmaschiges Netzwerk bilden, im Ansehen einem Texturboden nicht unähnlich. Bodentypologisch ist die ganze Schicht als $\mathrm{fB}-$ Horizont einer fossilen Parabraunerde anzusprechen. Der braune (10 YR 5/6) feinsandige Lehm ist von zahlreichen kleinen, schichtungslos verteilten Steinen durchsetzt und hat ein gut ausgebildetes Polyedergefüge. Blauschwarze Überzüge auf den Kluftflächen sind sehr gemein, rostbraune bis rostrote $(\mathrm{Fe}-)$ Flecken von $0,5-1 \mathrm{~cm} \phi$ sind hingegen sehr selten, und Konkretionen und Bleichungen wurden überhaupt nicht beobachtet. Nadelstichporen sind sehr zahlreich, größere Hohlräume (Wurzelröhren u. a.) haufig. 
Auf dem hangenden Solifluktionslöß, der stellenweise 2 Meter Mächtigkeit erreicht und in mehrere Substraten unterteilt werden kann, ist die spätglazial-holozäne Parabraunerde-Braunerde zur Entwicklung gelangt. Hinsichtlich der petrographischen Zusammensetzung unterscheidet sich dieser gräulich-braune (10 YR 4/4) feinsandige Lehm von den basalen wesentlich dadurch, daß er auch größere Steine (darunter angeätzte Kalke) enthält. Dies deutet darauf hin, daß zur Bildungszeit des oberen Fließlösses die solifluidale Denudation auf den höher gelegenen Teilen der Moräne bereits die interglaziale Braunlehmdecke bis auf den kalksteinhaltigen B/C-Horizont abgetragen hatte. Der deutlich höhere Tongehalt des vergleichsweise geringmächtigen basalen Lösses dürfte daher nur zum Teil Folge der im Vergleich zur rezenten intensiveren bzw. weiter fortgeschrittenen Pedogenese sein, in gewissem Umfange bereits als Bodenrelikt in den Löß hineingekommen sein.

Ein Gegenstück zu dem vorstehend beschriebenen interstadialen Lößboden auf der Rißmoräne am F. Chiese ist eine Bodenbildung in dem „Volpino" genannten Löß auf der Ostseite des Amphitheaters. Den besten Aufschluß bietet ein "Torriòn“ genannter Pfeiler in der tiefeingeschnittenen Valsorda, die von Bardolino nach E zum Monte Moscal hinaufführt.

Der tiefste im Tal heute noch zugängliche Boden ist ein rötlicher (5-7,5 YR 4/4) Braunlehm, der nach allen seinen petrographischen und typologischen Eigenschaften nur als letztinterglazial angesprochen werden kann (FRÄNZLE 1959, 1960). Nach oben geht dieser Plastosol in einer rund $50 \mathrm{~cm}$ mächtigen kryoturbaten Durchmischungsschicht in den hangenden Löß über; sein Stein- und Grusgehalt nimmt ab und die Farbe verliert an L.euchtkraft.

Das eigentliche Lößprofil des Torriòn setzt sich aus folgenden Schichten zusammen: $620-580 \mathrm{~cm}$ mittelbrauner (10 YR 4/3) humoser feinsandiger Lößlehm mit zahlreichen vergrusenden Steinen und Einzelmineralien, sehr dicht gelagert;

$580-480 \mathrm{~cm}$ dunkelgraubrauner bis olivgrauer $(2,5-5 \mathrm{Y}$ 4/2) humoser Lößlehm mit nach oben breiter werdenden Spaltenfüllungen, die im Anschnitt Ahnlichkeit mit Suturen haben und durch Fe-Verbindungen bald gelblichweiß, bald rostig gefärbt sind; auch punktförmige Rostflecken treten gelegentlich auf.

$480-300 \mathrm{~cm}$ tiefdunkel-graubrauner (10 YR - 2,5 Y 3/2) humoser Lößlehm mit sehr zahlreichen stäbchenförmigen $\mathrm{CaCO}_{3}$-Sekretionen;

$300-150 \mathrm{~cm}$ tiefdunkel-graubrauner (wie oben) humoser Lößlehm mit sehr schwach ausgeprägter Rostfleckigkeit ohne $\mathrm{CaCO}_{3}$-Sekretionen.

Das Gefüge des gesamten Profils ist schwach polyedrisch; darüber hinaus zeichnen sich die Randpartien - insbesondere die Südwand - durch eine außerordentlich großprismatische Absonderung aus, die schon von weitem sichtbar ist. Der Kalkgehalt schwankt innerhalb des Profils zwischen $0 \%$ (Basis) und $4,6 \%(350 \mathrm{~cm})$ und steigt an der Obergrenze infolge sekundärer Aufkalkung sprunghaft auf rd. $42 \%$ an. Bemerkenswert niedrig ist der Tongehalt des Lößlehms: er schwankt zwischen 4,3 und 7,8\%, wenn man von der kryoturbat gestörten Basisschicht absieht.

Die stratigraphische Stellung des Lösses und der daraus hervorgegangenen BodenBildung ergibt sich aus den Lagerungsverhältnissen. $\mathrm{Da}$ er dem letztinterglazialen Plastosol auflagert, ist er eindeutig würmeiszeitlich; da er von der äußersten Würmmoräne überlagert wird, dürfte er schon vor der Zeit der maximalen Temperaturabsenkung gebildet worden sein. Es ist nur logisch, dieses Temperaturminimum der Würmeiszeit auch in den Südalpen im späten Mittelwürm zu suchen. Dann bleibt aber für die Ablagerung des Lösses nur eine frühe Phase dieses Mittelwürms oder aber das Frühwürm.

Die Tatsache, daß das ganze Lößprofil des Torriòn mit Ausnahme der untersten steindurchsetzten 40 Zentimeter ziemlich stark humos ist, beweist, daß es sich um eine alloch- 
thone Bildung handelt, d. h. ein Bodensediment aus (ausschließlich oder vorherrschend) A-Horizont-Material. Im einzelnen lassen sich folgende Phasen der Lößsedimentation und Bodenbildung auf Grund der geschilderten Profileigenschaften ableiten:

1. Uberdeckung des Altmoränengebietes mit Löß.

2. Verlehmung in der folgenden Wärmephase.

3. Neuerliche - wohl kurzfristige - Kältephase, in der dieser braune Lehm mit dem liegenden letztinterglazialen Lehm durch kryoturbate Vorgänge vermischt und dann von einer Lößlage bedeckt wurde.

4. Wärmere Phase, in deren Verlauf der Löß zu einer Art Schwarzerde verwitterte.

5. Kühl-feuchte Phase (Frühphase der maximalen Gletscherausbreitung?), während der die Schwarzerde abgetragen und im Taltiefsten abgelagert wurde.

\section{B. Das Bildungsklima der fossilen Böden}

Uberblickt man die geschilderten Bodenbildungen, so stellt man fest, daß bislang keine rötlich gefärbten (nicht rubefizierte!) B-Horizonte, wie sie aus Mitteleuropa in stellenweise erheblicher Mächtigkeit (z. B. REMY 1960) bekannt geworden sind, in Italien angetroffen wurden. (Ein rot gefärbter Boden aus Löß im Becken von Leffe-Bergamo, den Herr Prof. MANCINI aus Florenz anläßlich des Bodenkundlichen Colloquiums vom 20. 7. 1960 in Bonn im Lichtbild demonstrierte, gehört möglicherweise dem letzten Interglazial an.) Rubefizierung tritt nur bei spätglazial-holozänen Böden der Braunerdeklasse auf Niederterrassenschottern auf, wo es infolge der extremen pedogenetischen Trockenheit zu scharfer sommerlicher Dehydratation kommt. Auf allen anderen Substraten kommt es rezent $\mathrm{zu}$ einer normalen Braunerde- und Parabraunerdebildung mit z. T. starker Versauerung sowie zur Pseudovergleyung.

Nach dem bis jetzt vorliegenden Material scheint es bei den rezenten Böden so zu sein, daß nach Osten zu infolge gesteigerter makroklimatischer Trockenheit und damit einhergehender geringerer Durchfeuchtung die Tendenz zur Pseudovergleyung abnimmt. Bei der schon frühzeitig (PENCK 1882) erkannten Parallelität der heutigen und der eiszeitlichen Niederschlagsverhältnisse ist damit zu rechnen, daß Ähnliches auch für die Interstadiale gilt. Die beschriebenen fossilen Böden passen sich diesem erwarteten Bild ein; aber es bedarf noch eingehender Untersuchungen, ehe sich entscheiden läßt, ob sie wirklich repräsentativ für regionale pedogenetische Zusammenhänge sind.

Einer besonderen Erwähnung bedürfen in diesem Zusammenhang die bei den Profilen 2 und 3 erwähnten Bleichungszonen, welche in ihrer ersten Anlage auf Frostspalten zurückgehen. Wie an anderer Stelle (FRÄNZLE 1960) ausführlicher dargestellt wurde, gehen sie mit aller Wahrscheinlichkeit bei dem Pseudogley aus Rißlöß auf einen Dauerfrostboden zurück, während dies für die würmzeitlichen Spaltennetze wegen der sehr viel geringeren Dimensionen nicht mit dem gleichen Wahrscheinlichkeitsgrad zu erschließen ist.

Ein Vergleich der rezenten Parabraunerden mit den fossilen Pseudogleyen zeigt dort, wo sie unter gleichen Bildungsbedingungen entstanden sind, daß der typologische Unterschied nur durch ein im Vergleich zum heutigen wesentlich ungünstigeres (kühlfeuchtes) Klima erklärt werden kann. Bei den anderen fossilen Böden spricht der im Vergleich zu den rezenten auf gleichem Muttergestein entweder geringere (Profil 5) oder höhere Verwitterungsgrad (Profil 4) für dementsprechende Unterschiede des Bildungsklimas: Im ersten Falle beschränkte sich die Pedogenese im wesentlichen auf Kalkabfuhr, im letzteren kam es sogar zur Tonverlagerung. Insgesamt liegen also die nämlichen klimabedingten Unterschiede zwischen fossiler (interstadialer) und rezenter Verwitterung vor, die bei der Erforschung mittel- und westeuropäischer Lößprofile gefunden wurden. 


\section{Zur Frage der stratigraphischen Einordnung der fossilen Böden}

Die vorstehend gemachte Feststellung führt endlich zur Frage nach dem stratigraphischen Aussagewert der beschriebenen Bodenbildungen und Bodensedimente.

Sicher ist die stratigraphische Stellung der in regionaler Verbreitung auftretenden früh-würmzeitlichen Fließerde, bzw. des ihr klimageschichtlich äquivalenten Kryoturbationshorizontes. Im Basaltteil bestehen beide aus interglazialem, manchmal extrem pseudovergleytem Plastosol und gehen nach oben in Solifluktions- bzw. Kryoturbationslöß3 über.

Im Gegensatz zu dieser überall anzutreffenden Stadialbildung ist die Anzahl der bis jetzt gefundenen interstadialen Bodenbildungen im Vergleich zur Größe des Gebietes noch sehr gering. $\mathrm{Da}$ außerdem die Profilausbildung auf engem Raum wechselt, bedarf es noch der Untersuchung möglichst vieler Aufschlüsse innerhalb der einzelnen Teillandschaften Oberitaliens, um das (regionale) Normal-Lößprofil zu erhalten.

Immerhin sind aber durch die mitgeteilten Beobachtungen (die zur Zeit durch chemische und mineralogische Untersuchungen ergänzt werden) für die letzte Eiszeit mindestens zwei, durch ein Interstadial getrennte Lößphasen nach der frühglazialen Fließerdezeit nachgewiesen. Auch sie werden ihrerseits wieder - wenn die in einigen Profilen angetroffenen Verhältnisse verallgemeinert werden dürfen - jeweils durch eine frühstadiale Solifluktions- bzw. Kryoturbationsphase eingeleitet. Es liegt also auch in Oberitalien die von BüDEL (1950) für die Gesamtheit der Würmeiszeit, von Woldstedt $(1956,1958)$ für die einzelnen Stadiale erkannte charakteristische klimatische Abfolge vor. Es steht zu hoffen, daß es gelingt, die einzelnen Solifluktions-, L.̈ß- und Bodenbildungsphasen durch weitere Detailforschung zu parallelisieren und zeitlich einzuordnen, um so schließlich zu einer der mittel- und westeuropäischen vergleichbaren Gliederung der Würmeiszeit zu gelangen.

\section{Verzeichnis der im Text angeführten Literatur:}

BECK, P.: Zur Revision der Quartärchronologie der Alpen. - Verh. d. III. Internat. Quartär-Konferenz, Wien 1938.

BüDEL, J.: Die Klimaphasen der Würmeiszeit. - Naturw. 37, 1950.

FräNZLE, O.: Untersuchungen über Ablagerungen und Böden im eiszeitlichen Gletschergebiet Norditaliens. - Erdkunde 13, 1959. - - Bemerkungen zur Gliederung und Paläoklimatologie des oberitalienischen Pleistozäns, insbesondere des Gardasee-Gebietes. - Erdkunde 14, 1960 (im Druck).

Habbe, K. A.: Zur klimatischen Morphologie des Alpensüdrands - Untersuchungen in den Moränenamphitheatern der Etsch und des Gardasees. - Nachr. Akad. Wiss. Göttingen, math.-phys. Kl., Jg. 1960, Nr. 10.

LEvy, Fr.: Die eiszeitliche Vergletscherung der Südalpen zwischen Dora Ripária und Etsch. - Z. f. Gletscherkunde 9, 1914/15.

Mensching, H.: Die Moränenlandschaft der Dora Ripária. - Veröff. Akad. f. Raumforschung und Landesplanung 28, (Mortensen-Festschrift) Hannover 1954.

Nangeroni, G.: Probabili tracce di morene würmiane stadiarie negli anfiteatri del Verbano e del Ceresio. - Milano 1954 (d) - - I terreni pleistocenici dell' anfiteatro morenico del Verbano e del territorio varesino. - Atti Soc. ital. Sci. nat. 93, 1954 (b).

Nicolis, E.: Depositi quaternari ne! Veronese. - Atti R. Ist. Veneto di Sci., Lettere ed Arti 7, VI, 1895.

Penck, A.: Die Vergletscherung der deutschen Alpen. Leipzig :

Penck, A. \& BrüCKner, E.: D: Alpen im Eiszeitalter, Bd. 3. Leipzig 1909.

REMY, H.: Die zeitliche Steilung der Rodderbergtuffe im rheinischen Löß. - Decheniana 112, Bonn 1960.

SACCo, F.: Sulla costituzione geologica degli altipiani isolati di Fossano, Salmour e Banale. Annali R. Acad. Agric. Torino 29, 1886. - - I terreni quaternari della collina di Torino. - Atti Soc. itaı. Sci. nat. 30, 1887.

SchöNhals, E.: Äolische Jungwürmablagerungen im zentr in alpinen Vereisungsgebiet. - Vortrag auf der Tagun: ${ }^{\prime}$. Deutschen Quarti 15.-17. 8. 1958 . 
Venzo, S.: Geomorphologische Aufnahme des Pleistozäns (Villafranchiano-Würm) im Bergamasker Gebiet und in der östlichen Brianza: Stratigraphie, Paläontologie und Klima. Geol. Rdschau. 40, Stuttgart 1952. - - Stadi della glaciazione del „Donau“ sotto al Günz nella serie lacustre di Leffe (Bergamo-Lombardia). - Geol. Bavar. 19, München 1953.

Viglino, A. \& Capeder, G.: Communicazione preliminare sul loess piemontese. - Boll. Soc. geol. ital. 17, Rom 1898.

WaLdbaur, H.: Zur Karte "Landformen im mittleren Europa“ $1: 2$ Mill. - Wissensch. Veröff. d. Deutsch. Inst. f. Länderkde. 15/16, 1958.

Woldstedt, P.: Ưber die Gliederung der Würmeiszeit und die Stellung der Lösse in ihr. - Eiszeitalter und Gegenwart 4/5, 1956. - - Eine neue Kurve der Würm-Eiszeit. - Ebda. 9, 1958.

Manuskr. eingeg. 15. 10. 1960.

Anschrift des Verf.: Dr. Otto Fränzle, Geograph. Institut der Univ., Bonn, Franziskanerstraße 2. 